\title{
Diurnal behaviour of the ionospheric Alfvén resonator signatures as observed at high latitude observatory Barentsburg $(L=15)$
}

\author{
N. V. Semenova and A. G. Yahnin \\ Polar Geophysical Institute, Apatity, 184209, Russia \\ Received: 3 January 2008 - Revised: 19 March 2008 - Accepted: 24 April 2008 - Published: 5 August 2008
}

\begin{abstract}
The signature of the ionospheric Alfvén resonator (IAR), so called spectral resonant structures (SRS) in the spectra of the electromagnetic noise in the range of 0.1$10 \mathrm{~Hz}$ is rather frequently observed with the search coil magnetometer at observatory Barentsburg on Svalbard $(L=15)$. In this report we discuss some peculiarities of diurnal occurrence of SRS at this high latitude station. We show that the pronounced minimum of the SRS occurrence around noon can not be explained by the diurnal variations of the solar zenith angle (illumination of ionosphere). We conclude that the SRS occurrence minimum is the result of the enhanced variability of ionospheric parameters when the observing point enters (during the Earth's rotation) the region of the ionospheric projection of the dayside cusp and its vicinity.
\end{abstract}

Keywords. Ionosphere (Polar ionosphere; Wave propagation) - Radio science (Electromagnetic noise and interference)

\section{Introduction}

Resonant structures observed in spectra of the geomagnetic field fluctuations (noise) in the range of $0.1-10 \mathrm{~Hz}$ are attributed to signatures of the ionospheric Alfvén resonator (IAR) (e.g. Polyakov and Rapoport, 1981). These spectral resonant structures (SRS) have been detected and investigated at low latitudes (Bösinger et al., 2002), at mid-latitudes (Belyaev et al., 2000; Pokhotelov et al., 2003; Odzimek et al., 2004; Molchanov et al., 2004), and in the auroral zone (Belyaev et al., 1999; Semenova et al., 1999; Demekhov et al., 2000; Yahnin et al., 2003; Hebden et al., 2005). Recently, SRS were observed on Svalbard, that is, at $L \sim 15$ (Semenova et al., 2005; Yeoman et al., 2007).

Correspondence to: N. V. Semenova

(nadezhda.semenova@gmail.com)
According to the IAR theory, properties of SRS are determined by the parameters of the ionosphere forming the IAR above the observing point. In particular, the maximal electron density in the F-region is an important parameter as well as the decay of the density above the F-region (Polyakov and Rapoport, 1981). In turn, the ionosphere characteristics are formed under influence of different factors; the significance of which for the IAR is different at different latitudes. At low latitudes the illumination by the Sun is the most important factor for the IAR formation. Thus, at $L=1.4$ SRS are always detected during nighttime, and never during daytime (Bösinger et al., 2002). Towards higher latitudes the influence of the solar illumination decreases since the elevation (zenith) angle of the Sun decreases. In the auroral zone, the particle precipitation and plasma convection are also important. Although the auroral ionosphere is illuminated by the Sun during summer time, the probability of observing SRS around local midnight is not negligible here (Yahnin et al., 2003). In winter, that is, during the "polar night", the probability of observing SRS is significant even at noon. In contrast to low latitudes, the probability of observing SRS in the auroral zone is less than $100 \%$ even in the darkest times. This is, in particular, due to geomagnetic activity; in the auroral zone the SRS occurrence decreases as geomagnetic activity increases. Such a dependence can be related to the increase of the conductivity in the E-region due to the precipitation of more energetic particles. The enhanced E-region conductivity leads to decrease of the IAR Q-factor (Trakhtengerts et al., 2000). Another reason that prevents the SRS detection in the auroral zone can be an enhanced variability of the ionosphere. Indeed, because of the low intensity and noisy nature, SRS are detectable only when resonance frequencies are relatively stable for long $(10-20 \mathrm{~min})$ time periods. This time has been estimated by many authors (e.g. Belyaev et al., 1999; Molchanov et al., 2004; Odzimek et al., 2004; Hebden et al., 2005) as an integration time needed to extract the weak SRS signal from the noise background. If the IAR

Published by Copernicus Publications on behalf of the European Geosciences Union. 


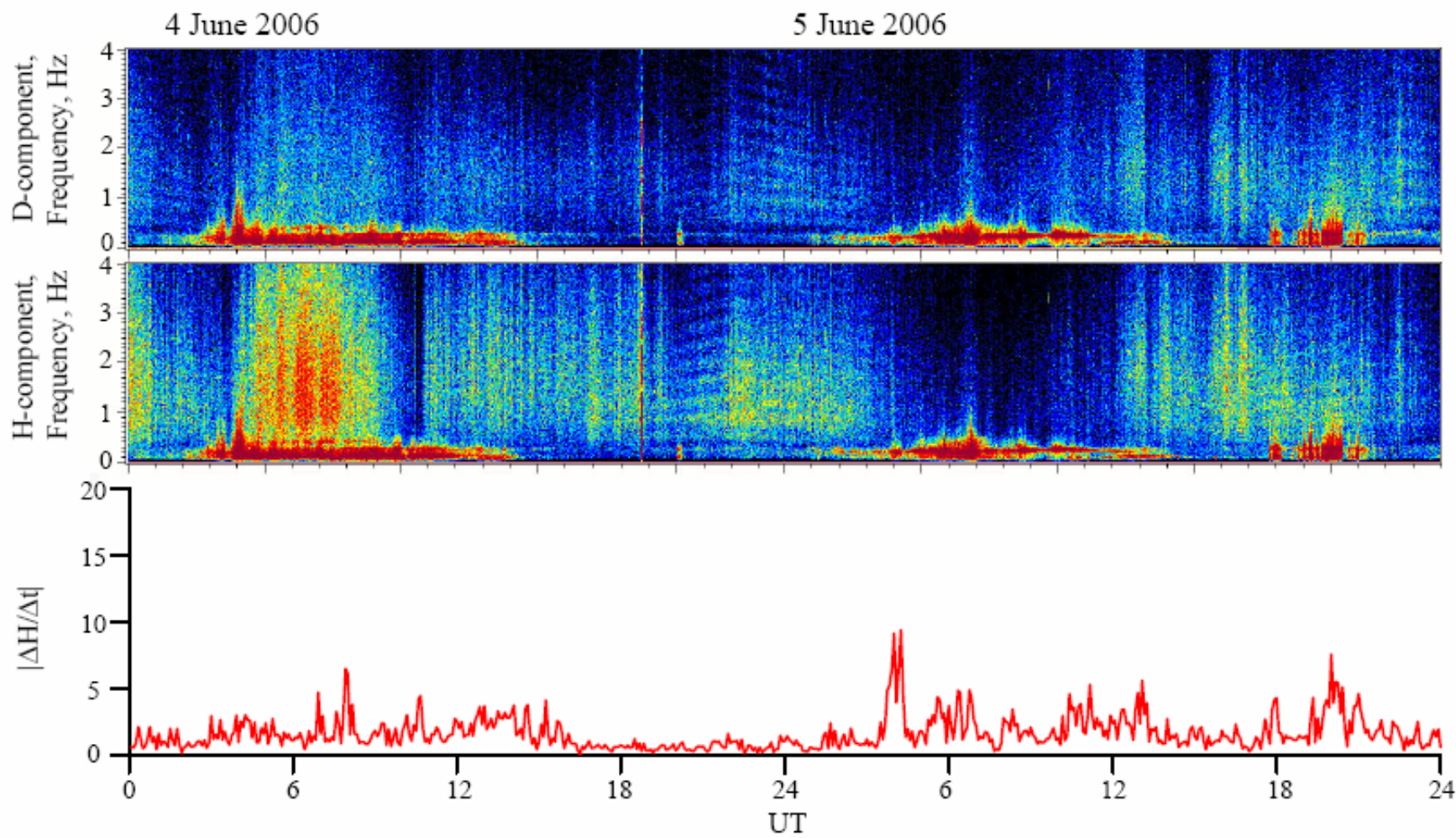

Fig. 1. Top: Spectrograms of $H$ and $D$ components of the geomagnetic fluctuations revealed from search coil magnetometer data in Barentsburg during two successive days (4 June 2006 and 5 June 2006). Bottom: Variations of the absolute value of the time derivative of the horizontal magnetic field obtained from the standard magnetometer measurements in Barentsburg for the same time interval.

properties significantly vary within this characteristic time, SRS can hardly be resolved in the dynamic spectra (spectrograms). The variability of ionospheric parameters, which is evident in the auroral zone during magnetic disturbances, prevents the formation of a stable IAR.

In this paper we discuss the possible reasons of the diurnal behaviour of SRS at higher latitudes based on observations in Barentsburg on Svalbard, where the sunlight effect is not expected to be significant. The next section will describe the observations. In Sect. 3 the result of calculations of the ionosphere reflection coefficient using a local ionospheric model based on EISCAT Svalbard radar data (Zhang et al., 2005) will be presented and compared with the observable features. In the Discussion section we will consider possible reasons for discrepancies between the observations and modelling.

\section{Diurnal behaviour of SRS and geomagnetic activity in Barentsburg}

The SRS observations have been carried out by the Polar Geophysical Institute at the geomagnetic observatory Barentsburg $\left(78.05^{\circ} \mathrm{N}, 14.12^{\circ} \mathrm{E}\right.$; Corrected Geomagnetic latitude (CGLat) is 75.17; MLT=UT+3) since 2002. The geomagnetic fluctuations are recorded by a search coil magne- tometer with a sampling rate of $40 \mathrm{~Hz}$. The instrument has a low-frequency cut-off at $0.05 \mathrm{~Hz}$ and a plateau-like amplitude response from $0.1 \mathrm{~Hz}$ up to tens of $\mathrm{Hz}$. The transformation factor of the instrument is $240 \mathrm{mV} / \mathrm{nT}$ at frequencies above $0.1 \mathrm{~Hz}$ and the sensitivity threshold is $<0.1 \mathrm{pT} / \mathrm{Hz}^{1 / 2}$ at $0.1 \mathrm{~Hz}$.

Upper panel in Fig. 1 presents a spectrogram of the magnetic fluctuations during two successive days (4-5 June 2006) in Barentsburg. The main feature, which we would like to emphasize, is the absence of SRS during the daytime hours. SRS are detected at 00:00-02:30 UT of 4 June, from 19:00 UT of 4 June till 04:00 UT of 5 June and at 17:00 24:00 UT of 5 June, that is, during the nighttime hours of MLT. The example in Fig. 1 relates to the summer, when the ionosphere above the observing point is illuminated by the Sun during the whole day. In Fig. 2 another spectrogram is shown, but for the winter season. The diurnal behaviour of SRS is, however, similar to that in Fig. 1. Such diurnal behaviour is statistically confirmed in Fig. 3 where the probability to observe SRS during the period of 2002-2006 is presented as dependent on UT. There were no days when SRS were observed within 09:00-12:00 UT (near noon) during the analyzed period. The probability of observing SRS increases during the nighttime hours. In this respect, the 


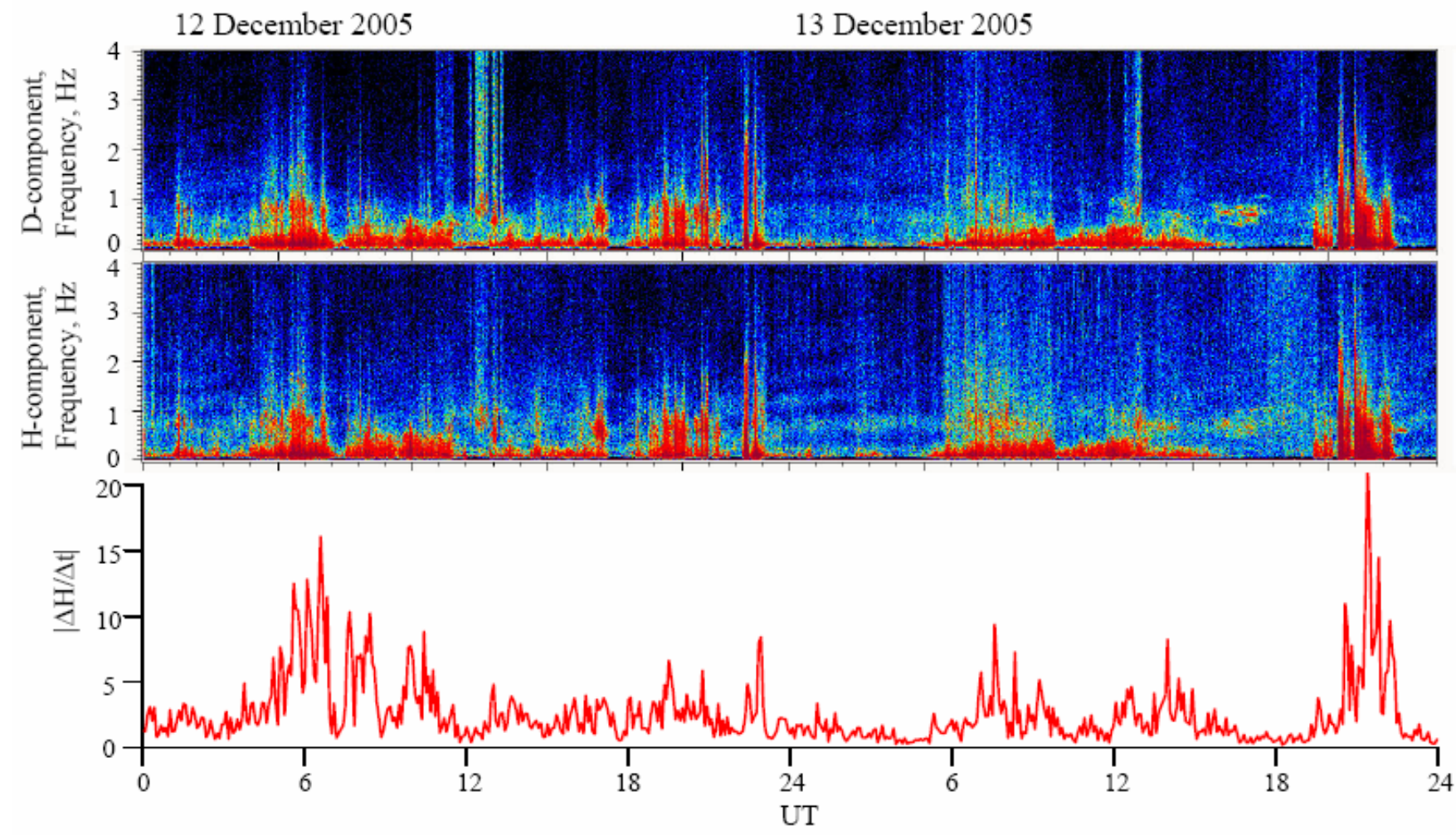

Fig. 2. The same as in Fig. 1 but for 12 December 2005 and 13 December 2005.

observations in Barentsburg resemble those at low latitudes (Bösinger et al., 2002), where the lack of SRS at daytime are, definitely, related to ionospheric illumination. However, the similar diurnal behaviour of SRS in any season as well as the considerations made in the following section, tell us that the illumination is not a controlling factor at such a high latitude.

The spectrograms presented in Figs. 1 and 2 demonstrate that around noon the magnetic fluctuations are enhanced in a wide frequency range. The search coil magnetometer allows us to resolve variations with periods less than $\sim 10 \mathrm{~s}$. A Bobrov type magnetometer in Barentsburg, which provides information on longer period variations, also shows an enhanced variability of the magnetic field near noon. The bottom panels of Figs. 1 and 2 represent the parameter characterizing the variability of geomagnetic field in Barentsburg, the $|\Delta H / \Delta t|$, where $H$ is the horizontal component of the ground magnetic field. As estimate we used values of $\Delta H$ taken for $\Delta t=5 \mathrm{~min}$ (other values of $\Delta t$ do not change the result qualitatively). It is clear from the comparison of the upper and bottom panels of Figs. 1 and 2 that SRS tend to appear when the variability of the magnetic field is small.

Yahnin et al. (2003) showed that probability of observing SRS in the auroral zone is higher during small values of the local $K$-index (characterizing the local amplitude of the magnetic field variation for a 3-h interval). The same is found for observations in Barentsburg. Indeed, the probability of

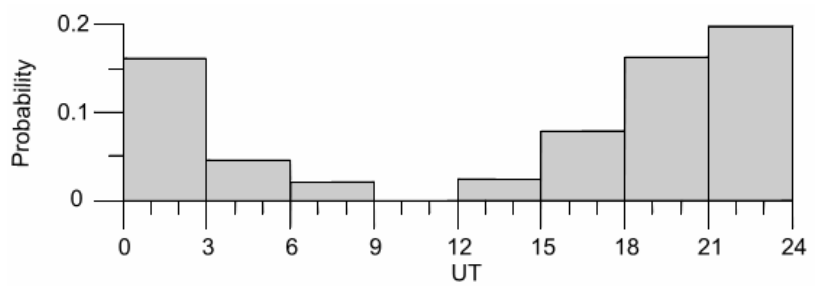

Fig. 3. The probability of observing the spectral resonant structures as a function of Universal Time revealed from the search coil magnetometer measurements in Barentsburg during 2002-2006.

observing SRS in Barentsburg is some $30 \%$ during $K=0$ and decreases with higher $K$-index (some $20 \%$ during $K=1,8 \%$ during $K=2$ ). During $K>2$ the probability is negligible.

The diurnal occurrence of magnetic disturbances with a $K$-index of a certain value in Barentsburg is shown in Fig. 4. For this plot the data for the year 2005 were used. From Fig. 4 it is clear that at any 3-h interval of the day the most probable value of $K$-index is 3 . The remarkable feature revealed from such a presentation of geomagnetic activity is that during the daytime there are only few days with $K=0$ 1. That is, around noon the magnetic field is almost always disturbed. 


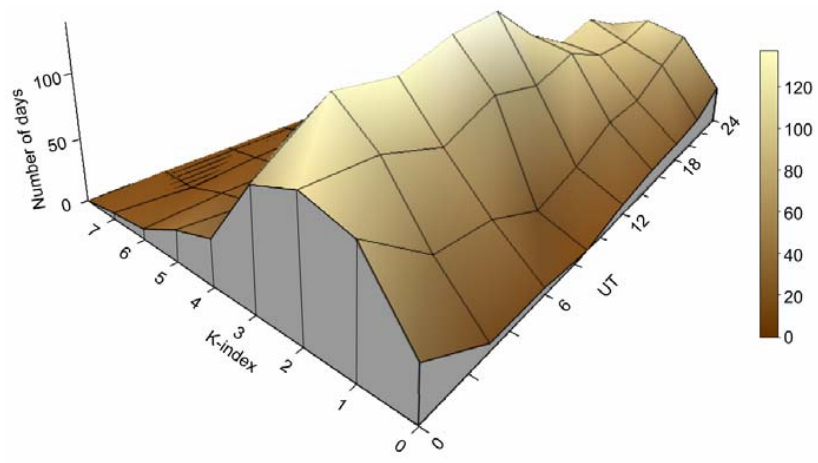

Fig. 4. The number of days in 2005 with a $K$-index of a certain value as function of the Universal Time. Note, that the lack of days with weak activity $K=0-1$ coincides with minimum of the SRS probability in Fig. 3.

\section{Modelling of the SRS occurrence}

The resonant structures can be recognized only if the background noise intensity varies significantly with frequency. According to the IAR theory, this occurs in the case of a large modulation of the upper ionosphere reflection coefficient as a function of frequency (or sufficiently large resonator quality, Q-factor). The larger the modulation, the more pronounced the SRS. The reflection coefficient can be calculated using an ionospheric model. The advantage of observations in Barentsburg is the close location of the EISCAT Svalbard radar. On the basis of this radar data a local ionospheric model has been constructed by Zhang et al. (2005). The model is a part of the Incoherent Scatter Radar Ionospheric Model (ISRIM) family, which has been based on data from the Madrigal distributed data system (http://www.openmadrigal.org). Using this model the average altitudinal profile of the electron density can be obtained for given date and time (which determine the Earth's dipole tilt and solar zenith angle, respectively). The solar activity level is characterized by index $F 10.7$, and geomagnetic activity level is characterized by the $a_{p}$-index.

We performed the calculations for four days representing different seasons (22 March, 22 June, 22 September, 22 December). To exclude influence of solar and geomagnetic activity we set values $F 10.7$ and $a_{p}$ as, respectively, 135 and 15 , representing median solar activity and weak geomagnetic activity. Thus, any variations in the modelled ionospheric profiles were due to changes of the solar zenith angle. First, diurnal variations of the height distribution of the electron density for selected days were obtained using the local ionospheric model. They are presented in Fig. 5 (on the left). Then, using the electron density height distributions the ionospheric reflection coefficient was calculated using the full wave algorithm of Ostapenko and Polyakov (1990). The result is shown in Fig. 5 (on the right) in the form of artificial spectrograms. All the calculated reflection coefficients have clear resonant struc- ture implying pronounced SRS at any local time. To characterize the depth of modulation of the reflection coefficient quantitatively one can introduce a parameter depending on the difference between the intensities of the coefficient in the minima and maxima. As such parameter we used $A=\left[\left(R_{12}-R_{1}\right)+\left(R_{12}-R_{2}\right)+\left(R_{23}-R_{2}\right)+\left(R_{23}-R_{3}\right)\right] / 4$, where $R_{1}, R_{2}, R_{3}$ are values of the reflection coefficient in first, second and third minima, and $R_{12}$ and $R_{23}$ are maximal values of the reflection coefficient between first and second minima, and between second and third minima, respectively. The diurnal behaviour of the parameter $A$ for different seasons exhibits no significant variations both with local time and season (not shown) implying equal chances for the SRS to be resolved. Thus, the calculations on the basis of the local ionosphere model demonstrate that the SRS observability is not significantly influenced by the ionospheric illumination by the Sun. This means that another reason must be responsible for the lack of dayside SRS observations. We suggest that this reason is related to the enhanced variability of the magnetic field observed at Barentsburg during the daytime hours.

\section{Summary and discussion}

In Sect. 2 we considered the occurrence of SRS at Barentsburg, Svalbard. The most remarkable feature in the diurnal behaviour of SRS is the total lack of SRS detection around noon, independent of the season. At the same time, the ground magnetic field at Barentsburg exhibits an enhanced variability near noon.

In Sect. 3 we modelled the influence of the illumination of the ionosphere above Svalbard on the ionospheric reflection coefficient during weak geomagnetic and median solar activity. A clear resonant structure is found in the reflection coefficient independent of UT and season, despite variations in the height distribution of the electron density. Although the IAR frequencies vary with time and season, estimates of the IAR quality demonstrate that SRS should be detectable during the whole day. It is clear, however, that the ionospheric model which is used for calculations can reproduce only average ionospheric parameters, but not their variability.

The IAR theory (e.g. Polyakov and Rappoport, 1981; Trakhtengerts et al., 2000) predicts, in particular, the dependence of the modulation depth of the reflection coefficient on two parameters characterizing the upper ionosphere: the electron density in the F-region and the scale of the electron density decay above the F-region maximum. The increase (decrease) of every of these parameters leads to the decrease (increase) of the modulation depth. An inspection of the electron density altitudinal profiles revealed from the model by Zhang et al. (2005) and used for the construction of Fig. 5 (left side) demonstrated that there is a general anti-correlation of these two parameters during their diurnal 


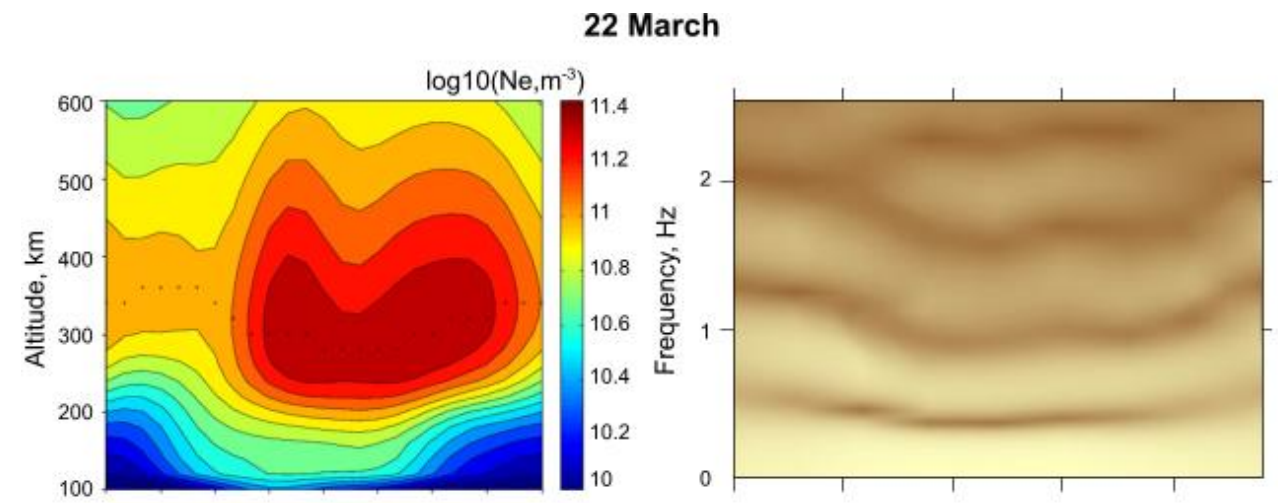

\section{June}
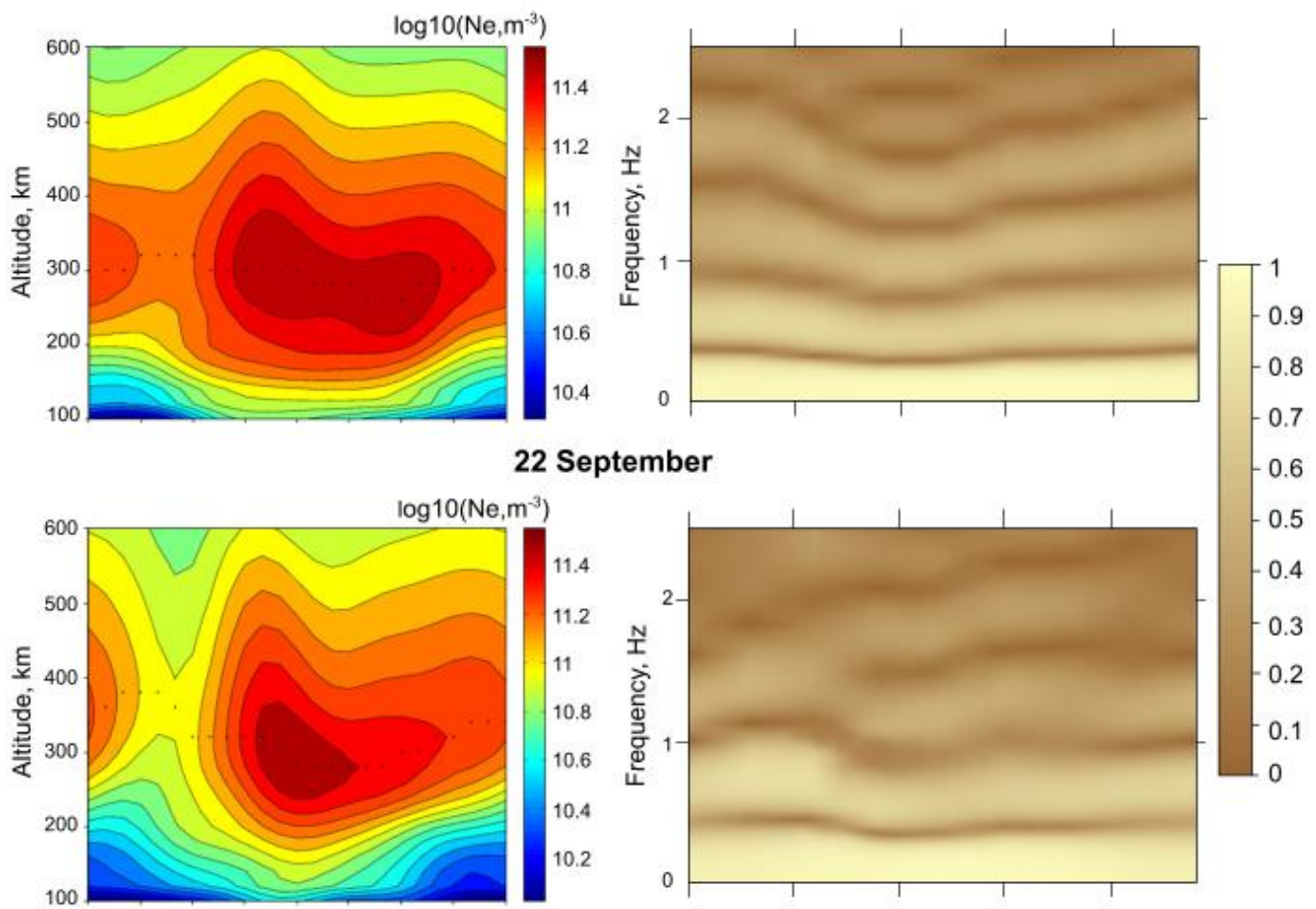

22 December
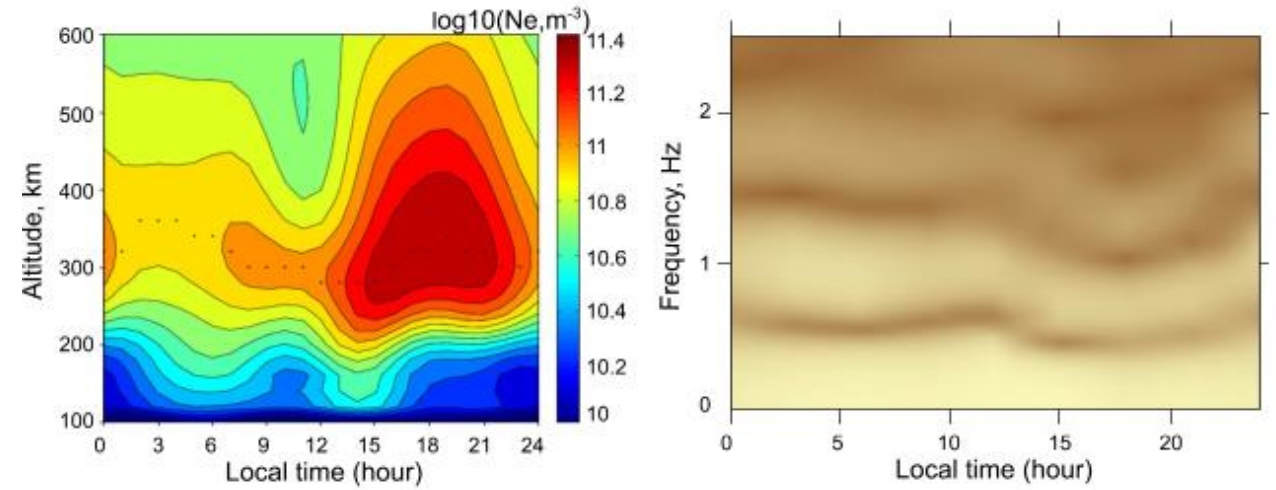

Fig. 5. On the left: Model representation of the diurnal variations of the electron density versus height above Svalbard. On the right: Diurnal variations of the ionospheric reflection coefficient calculated on the basis of the IAR theory, taking account of the electron density model. 
variations (not shown). Evidently, this should lead to relative stability of the SRS modulation depth in our modelling.

Viljanen et al. (2001) have statistically investigated the time derivative of the ground magnetic field variations using the IMAGE network of magnetometers in Fennoscandia and Svalbard. They showed that on Svalbard the number of timesteps with $|d H / d t|>1 \mathrm{nT} / \mathrm{s}$ has a pronounced local maximum around noon (their Fig. 8). This agrees with our conclusion on the dayside enhancement of the magnetic field variability on Svalbard.

The magnetic variability measured locally is, of course, not a direct measure for the ionospheric variability overhead. In particular, on the night side the magnetic disturbances can be produced by a substorm developing well equatorward of the high latitude station. In such cases the magnetic disturbances do not affect the SRS observability (see, for example, interval of 17:00-21:00 UT on 5 June 2005 in Fig. 1). It is worth noting that around noon Svalbard is typically in a region where several magnetospheric domains (such as cusp, plasma mantle, boundary layers) have their footprints. These domains (and their ionospheric counterparts) have different properties since they are populated with different kinds of particles, and their boundaries are rather unstable since they are subjected to the influence of the variable IMF and solar wind. The enhanced variability of the ground magnetic field in this region agrees with some statistical studies based on satellite measurements. For example, Matsuo et al. (2003) using the DE-2 satellite data stressed that the largest ionospheric electric field variability is observed in the vicinity of the cusp. Also, Shepherd et al. (2003) noted the high level of the ionospheric convection variability near the cusp. It is reasonable to consider the variability of the ground magnetic field as a manifestation of the variability of the ionosphere parameters.

From the CHAMP satellite data Ritter et al. (2004) found that during quiet periods (preferable conditions for the SRS observations) the strongest field aligned currents concentrate near the cusp region. Ritter et al. (2004) also noted that during such quiet periods the ionospheric Hall currents in the polar region are also enhanced around noon. One can expect that changes in the intensity and location of the currents (due to the variability of the interplanetary medium parameters) will produce a magnetic effect on the ground, which is larger at noon in comparison with other MLTs.

According to the fact that on the dayside the magnetic (and ionospheric) variations are seen at different time scales including periods of less than 10-20 min (see Figs. 1 and 2), the ionosphere above Barentsburg around noon is not stable in the sense of producing the conditions for the formation of stable IAR eigenfrequencies. This should prevent the SRS observations in this region. At other MLTs the station is situated poleward of the statistical auroral oval where one can expect a more stable ionosphere.

\section{Conclusion}

From the above considerations of the diurnal behaviour of SRS in Barentsburg it is revealed that there is clear dayside minimum of SRS occurrence at the latitudes of Svalbard, and this minimum can not be explained by the variations of the solar zenith angle. We conclude that impossibility of the SRS observations on Svalbard at the daytime relates to the enhanced variability of the ionosphere above the observing point, which is manifested by the variability of the ground magnetic field. We suggest that this enhanced variability indicates the entering of the observing point into the vicinity of the cusp, while at other MLTs Svalbard is situated poleward of the auroral oval where ionosphere is relatively stable.

We would like to emphasize the advantage of the SRS observations in Barentsburg in the close co-location with the Svalbard EISCAT radar. This opens the possibility to compare observed SRS characteristics with direct measurements of ionospheric parameters. This will be a task for future investigations.

Acknowledgements. We thank A. N. Vasilyev and other members of the engineering group in PGI as well as the staff of the observatory Barentsburg for good quality of the search coil magnetometer data. We thank members of the Haystack Observatory Atmospheric Sciences Group for assembling and maintaining the Madrigal database. We thank S.-R. Zhang and other colleagues responsible for developing the local ionospheric models and making them open for the community. The work was performed in frames of the basic research program \#16, part 3 of the Presidium of the Russian Academy of Science "Solar activity and physical processes in the Sun-Earth system" and the program of the Division of Physical Sciences of RAS "Plasma processes in the solar system". The study was supported by Russian Foundation for Basic Research (RFBR) through the grant \# 05-05-64212. N.V. Semenova thanks the organizing committee of the 13th EISCAT Workshop as well as RFBR for the support of her participation in the meeting where this report has been presented.

Topical Editor K. Kauristie thanks T. Yeoman and another anonymous referee for their help in evaluating this paper.

\section{References}

Belyaev, P. P., Bosinger, T., Isaev, S. V., Trakhtengerts, V. Y., and Kangas, J.: First evidence at high latitude for the ionospheric Alfvén resonator, J. Geophys. Res., 104(A3), 4305-4318, 1999.

Belyaev, P. P., Polyakov, S. V., Ermakova, E. N., and Isaev, S. V.: Solar cycle variations in the ionospheric Alfvén resonator 19851995, J. Atmos. Solar-Terr. Phys., 62, 239-248, 2000.

Bösinger, T., Haldopis, C., Belyaev, P. P., Yakunin, M. N., Semenova, N.V., Demekhov, A. G., and Angelopoulos, V.: Spectral properties of the ionospheric Alfvén resonator as observed at a low latitude station (L=1.3), J. Geophys. Res, 107(A10), 1281, doi:10.1029/2001JA005076, 2002.

Demekhov, A. G., Belyaev, P. P., Isaev, S. V., Manninen, J., Turunen, T., and Kangas, J.: Modelling diurnal evolution of the resonance spectral structure of the atmospheric noise background in 
the Pc 1 frequency range, J. Atmos. Solar-Terr. Phys., 62, 257265,2000

Hebden, S. R., Robinson, T. R., Wright, D. M., Yeoman, T., Raita, T., and Bösinger, T.: A quantitative analysis of the diurnal evolution of Ionospheric Alfvén resonator magnetic resonance features and calculation of changing IAR parameters, Ann. Geophys., 23, 1711-1721, 2005, http://www.ann-geophys.net/23/1711/2005/.

Matsuo, T., Richmond, A. D., and Hensel, K.: High-latitude ionospheric electric field variability and electric potential derived from DE-2 plasma drift measurements: Dependence on IMF and dipole tilt, J. Geophys. Res., 108(A1), 1005, doi:10.1029/2002JA009429, 2003.

Molchanov, O. A., Schekotov, A. Yu., Fedorov, E., and Hayakawa, M.: Ionospheric Alfvén resonance at middle latitudes: results of observations at Kamchatka, Phys. Chem. Earth, 29, 649-655, 2004.

Odzimek, A.: Numerical estimate of the spectral resonance structure frequency scale of natural ULF magnetic field, Stud. Gephys. Geod., 48, 647-660, 2004.

Ostapenko, A. A. and Polyakov, S. V.: The dynamics of the reflection coefficient of Alfvén waves in Pc1 range from ionosphere under variation of electron concentration of the lower ionosphere, Geomagnetism and Aeronomy, 30(1), 50-56, 1990.

Pokhotelov, O. A., Feygin, F. Z., Khabazin, Yu. G., Khruschev, V. V., Bosinger, T., Kangas, J., and Prikner, K.: Observations of IAR spectral resonance structures at a large triangle of geophysical observatories, "Physics of Auroral Phenomena", Proc. XXII Annual Seminar, Apatity, 123-126, 2003.

Polyakov, S. V. and Rapoport, V. O.: Ionospheric Alfvén Resonator, Geomagnetism and Aeronomy, 21(5), 610-614, 1981.

Ritter, P., Luhr, H., Maus, S., and Viljanen, A.: High-latitude ionospheric currents during very quiet times: their characteristics and predictability, Ann. Geophys., 22, 2001-2014, 2004, http://www.ann-geophys.net/22/2001/2004/.

Semenova, N. V., Yahnin, A. G., Titova, E. E., Kangas, J., Kultima, J., Manninen, J., and Turunen, T.: Statistical characteristics of the spectral resonance structures of electromagnetic background noise in the frequency range $0-4 \mathrm{~Hz}$ inferred from the auroral zone measurements, "Physics of Auroral Phenomena", Proc. XXII Annual Seminar, Apatity, 86-89, 1999.
Semenova, N. V., Yahnin, A. G., Vasiliev, A. N., Noskov, S. P., and Voronin, A. I.: First observations of the electromagnetic noise spectral resonance structures in the range of $0.1-4 \mathrm{~Hz}$ in the polar cap region (Barentsburg, Spitsbergen), in: Complex Investigations of Spitsbergen Nature, Kol'skiy Sci. Cent., Russ. Acad. of Sci., Apatity, Russia., 5, 120-131, 2005 (in Russian).

Shepherd, S. G., Ruohoniemi, J. M., and Greenwald, R. A.: Direct measurements of the ionospheric convection variability near the cusp/throat, Geophys. Res. Lett., 30(21), 2109, doi:10.1029/2003GL017668, 2003.

Trakhtengerts, V. Y., Demekhov, A. G., Belyaev, P. P., Polyakov, S. V., Ermakova, E. N., and Isaev, S. V.: Mechanism of anticorrelation in the occurrence of ULF electromagnetic noise resonance structure and Pc1 magnetic pulsations through the solar activity cycle, J. Atmos. Solar-Terr. Phys., 62, 253-256, 2000.

Viljanen, A., Nevanlinna, H., Pajunpaa, K., and Pulkkinen, A.: Time derivative of the horizontal geomagnetic field as an activity indicator, Ann. Geophys., 19, 1107-1118, 2001, http://www.ann-geophys.net/19/1107/2001/.

Yahnin, A. G., Semenova, N. V., Ostapenko, A. A., Kangas, J., Manninen, J., and Turunen, T.: Morphology of the Spectral Resonance Structure of the Electromagnetic Noise in the Range of $0.1-4 \mathrm{~Hz}$ at $L=5.2$, Ann. Geophys., 21, 779-786, 2003, http://www.ann-geophys.net/21/779/2003/.

Yeoman, T. K., Scoffield, H. C., Wright, D. M., Baddeley, L. J., Vasilyev, A. N., and Semenova, N. V.: Investigation of natural and artificial stimulation of the ionospheric Alfvén resonator at high latitude, J. Adv. Space Res., doi:10.1016/j.asr.2007.03.083, 2007.

Zhang, S.-R., Holt, J. M., van Eyken, A. P., McCready, M., AmoryMazaudier, C., Fukao, S., and Sulzer, M.: Ionospheric local model and climatology from long-term databases of multiple incoherent scatter radars, Geophys. Res. Lett., 32, L20102, doi:10.1029/2005GL023603, 2005. 